PROCEEDINGS OF THE

AMERICAN MATHEMATICAL SOCIETY

Volume 132, Number 8, Pages 2475-2481

S 0002-9939(04)07388-5

Article electronically published on March 25, 2004

\title{
INDIVIDUAL ERGODIC THEOREM FOR UNITARY MAPS OF RANDOM MATRICES
}

\author{
RYSZARD JAJTE
}

(Communicated by Andreas Seeger)

\begin{abstract}
Using simple techniques of finite von Neumann algebras, we prove a limit theorem for random matrices.
\end{abstract}

\section{Notation AND MAin RESUlt}

1.1. Let $(\Omega, \mathcal{F}, \mu)$ be a probability space, and let $\mathcal{H}$ denote the space of all $d \times d$ matrices with entries in the complex space $L_{2}(\Omega, \mu)$. $\mathcal{H}$ is a Hilbert space with the inner product

$$
\langle A B\rangle=\Phi\left(A B^{*}\right), \quad \text { where } \quad \phi(A)=\int_{\Omega} \operatorname{tr} A(\omega) \mu(d \mu),
$$

and tr denotes the normalized trace on the algebra $M_{d}$ of $d \times d$ complex matrices. A matrix $A$ with $\mathcal{F}$-measurable entries (random matrix) is said to be positive $(A \geq 0)$ if for $\mu$-almost all $\omega \in \Omega, A(\omega)$ is positive definite in $\mathbb{C}^{d}$, i.e., $(A(\omega) \lambda, \lambda) \geq 0$ for $\lambda \in \mathbb{C}^{d} . A \geq B$ means $A-B \geq 0$. A map $\alpha: \mathcal{H} \rightarrow \mathcal{H}$ is said to be positive if $\alpha A \geq 0$ for $A \geq 0$. Throughout the paper 1 denotes the unit random matrix, i.e., the diagonal matrix with the entries $a_{i k}=\delta_{i}^{k} \mu$-a.e.

The main goal of the paper is the following individual ergodic theorem.

1.2. Theorem. Let $\alpha$ be a positive unitary operator in $\mathcal{H}$, satisfying the condition

$$
\frac{1}{n} \sum_{r=-n}^{n} \alpha^{r} \mathbf{1} \leq Y, \quad n=1,2, \ldots,
$$

for some random matrix $Y$ with $\phi(Y)<\infty$.

Let us put

$$
S_{n}=\frac{1}{n} \sum_{k=0}^{n-1} \alpha^{k}
$$

Then, for each $A \in \mathcal{H}$,

$$
\left\|\left(S_{n} A\right)(\omega)-\tilde{A}(\omega)\right\| \rightarrow 0 \quad \mu \text {-a.e., }
$$

$\|\cdot\|$ being an arbitrary fixed norm in $M_{d}$; here $\tilde{A}$ is given by the mean ergodic theorem for $\alpha$.

Received by the editors August 12, 2002 and, in revised form, February 26, 2003.

2000 Mathematics Subject Classification. Primary 60F15, 46L10.

Key words and phrases. Random matrix, positive isometry, ergodic theorem, von Neumann algebra. 
Clearly, the above result is closely related to the well-known theorem of A. Ionescu Tulcea [6] (with improvements given by A. de la Torre 2] and Charn Huen Kan [1]). The proof of the classical individual ergodic theorem for a positive isometry in $L_{p}(\Omega, \mu)$ is based on the dominated ergodic estimate. Because of the noncommutative structure of $\mathcal{H}$ we have no possibility of defining the maximal function for the isometry $\alpha$ in $\mathcal{H}$. That is why we have to find another way to establish our result. In the proof we use some techniques borrowed from the theory of operator algebras ([12], [13], 7]). We also follow some ideas of R. Duncan ([4], [5]).

\section{Proof of The THEOREM}

2.1. Let $\mathcal{M}$ be the algebra of all $d \times d$ matrices with entries in $L_{\infty}(\Omega, \mu)$. Then $\mathcal{M}$ is a finite von Neumann algebra (under pointwise multiplication) with the tracial state $\phi: A \rightarrow \int_{\Omega} \operatorname{tr} A d \mu$. Clearly, we can write

$$
\mathcal{M}=L_{\infty} \otimes M_{d}
$$

$M_{d}$ being endowed here with the standard $C^{*}$-norm. Then, since $\phi\left(|A|^{2}\right)=$ $\sum_{i, k} \int_{\Omega}\left|a_{i k}\right|^{2} d \mu, \mathcal{H}=L_{2}(\Omega, \mu) \otimes M_{d}$ is nothing but $L_{2}(\mathcal{M}, \phi)$ - the noncommutative $L_{2}$-space over the von Neumann algebra $\mathcal{M}$ with the finite trace $\phi$. In such a context the random matrices from $\mathcal{H}=L_{2}(\mathcal{M}, \phi)$ act as (unbounded, in general) operators in the Hilbert space $K=L_{2}(\Omega, \mu) \otimes C^{d}$ of random vectors $\left(\alpha_{1}, \ldots, \alpha_{d}\right)$ with $\alpha_{j} \in L_{2}(\Omega, \mu)$. In the sequel we shall use some basic facts from the theory of noncommutative $L_{p}$-spaces ([3, , 9], [7]) in the simplest case of $L_{2}$ over a finite von Neumann algebra $\mathcal{M}$. In particular, it is known that the space $L_{2}(\mathcal{M}, \phi)$ consists of densely defined closed linear operators in $K$ affiliated to $\mathcal{M}$. Affiliation means that if $x=u a$ is a polar decomposition of $x \in L_{2}(\mathcal{M}, \phi)$, then the partial isometry $u$ and all spectral projections of $a$ belong to $\mathcal{M}$. In the sequel $\|\cdot\|_{2}$ denotes the norm in $\mathcal{H}$. The norm in $\mathcal{M}$ will be denoted by $\|\cdot\|_{\infty}$. A crucial point for us is the following important result.

2.2. Lemma ([12, p. 197]). If $T$ is a densely defined symmetric operator affiliated to a finite von Neumann algebra, then its closure $T$ is selfadjoint and it is the unique selfadjoint extension of $T$.

In the sequel we shall need one more lemma. Keeping the notation used in the theorem, we have the following.

2.3. Lemma (cf. [5], [11). For all sequences $\left(p_{k}\right)$ of mutually orthogonal projections in $\mathcal{M}$,

$$
\sum_{n} \phi\left(R_{n+1} S_{n+1}^{*}\left(p_{n+1}\right)\right)<\infty
$$

where $R_{n}=\sum_{k=1}^{n} S_{k}^{*}\left(p_{k}\right)$.

Proof. The proof is a modification of the argument in [5]. By Lemma 2.2, since $B_{m}=S_{m}^{*}\left(p_{m}\right) \geq 0$, the operators $B_{m}$ are positive selfadjoint (unbounded, in general). Taking, if necessary, bounded operators $B_{m}^{(k)}=B_{m} E_{B_{m}}[0, k]$, putting 
them under inner product and letting $k$ go to infinity, we get

$$
\begin{aligned}
\phi\left(R_{n+1} S_{n+1}^{*}\left(p_{n+1}\right)\right)=\sum_{k=1}^{n+1} & \left\langle S_{k}^{*}\left(p_{k}\right), S_{n+1}^{*}\left(p_{n+1}\right)\right\rangle \\
& =\sum_{k=1}^{n+1}\left\langle p_{k}, S_{k} S_{n+1}^{*}\left(p_{n+1}\right)\right\rangle=\sum_{k=1}^{n+1} \phi\left(p_{k} S_{k} S_{n+1}^{*}\left(p_{n+1}\right)\right\rangle .
\end{aligned}
$$

Moreover,

$$
S_{k} S_{n+1}^{*}\left(p_{n+1}\right)=\frac{1}{k(n+1)} \sum_{j=0}^{k-1} \alpha^{j} \sum_{i=0}^{n} \alpha^{-i}\left(p_{n+1}\right)=\frac{1}{k(n+1)} \sum_{r=-n}^{n} c_{r} \alpha^{r}\left(p_{n+1}\right)
$$

with some $c_{r}$ 's. It is easy to check that $c_{r} \leq k$. So we get

$$
0 \leq S_{k} S_{n+1}^{*}\left(p_{n+1}\right) \leq \frac{1}{n+1} \sum_{r=-n}^{n} \alpha^{r}\left(p_{n+1}\right)
$$

and, consequently,

$$
\phi\left(p_{k} S_{k} S_{n+1}^{*}\left(p_{n+1}\right) \leq \frac{1}{n+1} \sum_{r=-n}^{n} \phi\left(p_{k} \alpha^{r}\left(p_{n+1}\right)\right) .\right.
$$

Thus we have

$$
\begin{aligned}
\sum_{k=1}^{n+1} \phi\left(p_{k} S_{k} S_{n+1}^{*}\left(p_{n+1}\right)\right. & \leq \sum_{k=1}^{n+1} \frac{1}{n+1} \sum_{r=-n}^{n} \phi\left(p_{k} \alpha^{r}\left(p_{n+1}\right)\right) \\
& =\frac{1}{n+1} \sum_{r=-n}^{n} \phi\left(\sum_{k=1}^{n+1} p_{k} \alpha^{r}\left(p_{n+1}\right)\right) \\
& \leq \frac{1}{n+1} \sum_{r=-n}^{n} \phi\left(\alpha^{r}\left(p_{n+1}\right)\right) \leq \phi\left(\frac{1}{n+1} \sum_{r=-n}^{n} \alpha^{r}\left(p_{n+1}\right)\right) \\
& \leq \phi\left(p_{n+1} \frac{1}{n+1} \sum_{r=-n}^{n} \alpha^{r} \mathbf{1}\right) \leq \phi\left(p_{n+1} Y\right)
\end{aligned}
$$

So, finally,

$$
\sum_{n} \phi\left(R_{n+1} S_{n+1}^{*}\left(p_{n+1}\right)\right) \leq \sum_{n} \phi\left(p_{n+1} Y\right) \leq \phi(Y)<\infty,
$$

which completes the proof of the lemma.

2.4. Let $A \geq 0$ be a matrix in $\mathcal{H}$. We identify $A$ with an operator from $L_{2}(\mathcal{M}, \phi)$. Denote by $D_{A}$ the domain of $A$ as an operator on $K$, i.e., $D_{A}=\{h \in K ; A h \in K\}$. Then $\left(A, D_{A}\right)$ is closed, positive, symmetric, and affiliated to $\mathcal{M}$. Consequently, by Lemma 2.2, $A$ is positive selfadjoint. Then the $S_{n}(A)$ are positive selfadjoint in $K$. Let us put $q_{0}=0$. Define successively

$$
p_{n}=e_{(\epsilon, \infty)}\left\{\left(1-q_{n-1}\right) S_{n}(A)\left(1-q_{n-1}\right)\right\}, \quad n=1,2, \ldots,
$$

and $q_{n}=q_{n-1}+p_{n}$. Here and elsewhere, $e_{Z}\{B\}$ denotes the spectral projection of the (selfadjoint) operator $B$, corresponding to a Borel set $Z \in \mathbb{R}$. Clearly $\left(p_{n}\right)$ 
are mutually orthogonal projections and $q_{n}=p_{1}+\cdots+p_{n}$. Moreover, since $p_{k} \leq$ $1-q_{k-1}$, we have

$$
\begin{aligned}
\phi\left(p_{k} S_{k}(A)\right) & =\phi\left(p_{k}\left(1-q_{k-1}\right) S_{k}(A)\left(1-q_{k-1}\right) p_{k}\right) \\
& =\epsilon \int_{\epsilon}^{\infty} \frac{\lambda}{\epsilon} \phi\left(e_{d \lambda}\left\{\left(1-q_{k-1}\right) S_{k}(A)\left(1-q_{k-1}\right)\right\}\right. \\
& \geq \epsilon \phi\left(p_{k}\right) .
\end{aligned}
$$

Thus we have

$$
\begin{aligned}
\phi\left(q_{n}\right)=\sum_{k=1}^{n} \phi\left(p_{k}\right) \leq \frac{1}{\epsilon} \sum_{k=1}^{n} \phi\left(p_{k} S_{k}(A)\right) & =\frac{1}{\epsilon} \sum_{k=1}^{n}\left\langle S_{k}(A), p_{k}\right\rangle \\
= & \frac{1}{\epsilon}\left\langle A, \sum_{k=1}^{n} S_{k}^{*}\left(p_{k}\right)\right\rangle \leq \frac{1}{\epsilon}\|A\|_{2}\left\|\sum_{k=1}^{n} S_{k}^{*}\left(p_{k}\right)\right\|_{2} .
\end{aligned}
$$

By Lemma 2.3,

$$
\sup _{n}\left\|\sum_{k=1}^{n} S_{k}^{*}\left(p_{k}\right)\right\|_{2}=\sup _{n}\left\|R_{n}\right\|_{2}=L<\infty .
$$

Indeed, $\sum_{n} \Phi\left(R_{n+1}\left(R_{n+1}-R_{n}\right)\right)<\infty$ implies $\sum_{n} \Phi\left(R_{n}\left(R_{n+1}-R_{n}\right)\right)<\infty$, and $\sum_{n}\left\|R_{n}\right\|_{2}<\infty$ follows.

Thus we can write

$$
\phi\left(q_{n}\right) \leq \frac{L}{\epsilon}\|A\|_{2}=\frac{L}{\epsilon} \Phi\left(|A|^{2}\right)^{1 / 2}, \quad n=1,2, \ldots .
$$

Putting $1-q=\sum_{k=1}^{\infty} p_{k}$, we have

$$
\phi(1-q) \leq \frac{L}{\epsilon} \phi\left(|A|^{2}\right)^{1 / 2}
$$

and

$$
\left\|q S_{k}(A) q\right\|_{\infty} \leq\left\|\left(1-p_{k}\right)\left(1-q_{k-1}\right) S_{k}(A)\left(1-q_{k-1}\right)\left(1-p_{k}\right)\right\|_{\infty}<\epsilon,
$$

for $k=1,2, \ldots$, by definition of $p_{k}$.

Summing up, we get the following lemma.

2.5. Lemma. Let $0 \leq A \in \mathcal{H}=L_{2}(\mathcal{M}, \phi)$. Then for $\epsilon>0$, there is a projection $q \in \mathcal{M}$ such that

$$
\left\|q S_{k}(A) q\right\|_{\infty}<\epsilon, \quad k=1,2, \ldots,
$$

and

$$
\phi(1-q) \leq \frac{L}{\epsilon} \phi\left(|A|^{2}\right)^{1 / 2},
$$

for some positive constant $L$, depending only on $\alpha$.

For further convenience, let us formulate one more elementary lemma.

2.6. Lemma. For a sequence $\left(A_{n}\right) \subset \mathcal{H}$, the following two conditions are equivalent:

(i) $\left\|A_{n}(\omega)\right\| \rightarrow 0 \mu$-a.e., for any norm $\|\cdot\|$ in $M_{d}$;

(ii) for each $\epsilon>0$, there is a projection $p \in \mathcal{M}$ such that $A_{n} p \in \mathcal{M}, n=1,2, \ldots$, $\phi(1-p)<\epsilon$ and $\left\|A_{n} p\right\|_{\infty} \rightarrow 0$. 
Proof. (i) $\rightarrow$ (ii). By the Yegorov theorem, for each $\epsilon>0$ there is a set $Z \in F$ with $\mu(\Omega \backslash Z)<\epsilon$ and such that

$$
\sup _{\omega \in Z}\left\|A_{n}(\omega)\right\| 1_{Z}(\omega)<\infty, \quad n=1,2, \ldots,
$$

and $\sup _{\omega \in Z}\left\|A_{n}(\omega)\right\| 1_{Z}(\omega) \rightarrow 0$. This implies that, for the entries $a_{i j}^{(n)}$ of $A_{n}$, we have $a_{i j}^{(n)} 1_{Z} \in L_{\infty}(\Omega, \mu)$ and $\left\|a_{i j}^{(n)} 1_{Z}\right\|_{L_{\infty}} \rightarrow 0$ as $\mu \rightarrow \infty(i, j=1, \ldots, d)$. Putting

$$
p(\omega)= \begin{cases}1 & \text { for } \omega \in Z, \\ 0 & \text { for } \omega \notin Z,\end{cases}
$$

we get a projection $p \in \mathcal{M}$ satisfying (ii).

(ii) $\rightarrow$ (i). Let $\epsilon>0$ and let $p$ satisfy (ii). Then $p=p(\omega)$, with $p(\omega)$ being the projections in $M_{d}$. Clearly, $\operatorname{tr} p(\omega) \leq \frac{1-d}{d}$, for $p \neq \mathbf{1}$. Let $p(\omega)=\mathbf{1}$, for $\omega \in Z$. Then

$$
1-\epsilon<\phi(p) \leq \mu(Z)+\frac{d-1}{d}(1-\mu(Z)) .
$$

Consequently, $\mu(Z) \geq 1-\epsilon d$. Moreover,

$$
\underset{\omega \in \Omega}{\operatorname{ess} \sup }\left\|A_{n}(\omega) p(\omega)\right\|_{\infty} \rightarrow 0
$$

which implies

$$
\sup _{\omega \in Z}\left\|A_{n}(\omega)\right\| \rightarrow 0, \text { with } \mu(Z) \geq 1-d \epsilon .
$$

Since $\epsilon$ is arbitrary, (i) follows.

2.7. Having Lemmas 2.5 and 2.6, we can conclude the proof in a rather standard way.

Let us write

$$
\mathcal{H}=\mathcal{H}_{1} \oplus \mathcal{H}_{2},
$$

when

$$
\begin{aligned}
& \mathcal{H}_{1}=\{B \in \mathcal{H} ; \alpha B=B\}, \\
& \mathcal{H}_{2}=\{B-\alpha B ; B \in \mathcal{H}\}^{-} .
\end{aligned}
$$

Let $A \in \mathcal{H}$. Then $A=B_{1}+B_{2}$ with $B_{j} \in \mathcal{H}_{j}$. Clearly, $B_{1}=\tilde{A}$ - the limit of $S_{k}(A)$ in the mean ergodic theorem. Let $\epsilon>0$. We have

$A-\tilde{A}=B-\alpha B+b, \quad$ with $b=b_{1}-b_{2}+i\left(b_{3}-b_{4}\right), b_{i} \geq 0,\left\|b_{i}\right\|_{2}<\frac{\epsilon^{2}}{16 L}, i=1, \ldots, 4$,

where $L$ is taken from Lemma 2.5.

By the standard decomposition we can write $B$ as the linear combination

$$
B=D_{1}-D_{2}+i\left(D_{3}-D_{4}\right), \quad \text { with } \quad D_{i} \geq 0 .
$$

So without any loss of generality, we can assume $B \geq 0$. Let $B=\int_{0}^{\infty} \lambda e(d \lambda)$ (spectral decomposition). Then, for $c$ large enough, we have

$$
\begin{aligned}
B=\int_{0}^{c} \lambda e(d \lambda)+d & =D+d, \quad \text { with } \quad\|D\|_{\infty} \leq c \\
\text { and } \quad d & =d_{1}-d_{2}+i\left(d_{3}-d_{4}\right), d_{i} \geq 0, \quad\|d\|_{2}<\frac{\epsilon^{2}}{16 L}, \quad i=1, \ldots, 4 .
\end{aligned}
$$


Let us remark that

$$
\sum_{k} \phi\left(\left|\frac{1}{k} \alpha^{k} D\right|^{2}\right)=\sum_{k} \frac{1}{k^{2}} \phi\left(\left|\alpha^{k} D\right|^{2}\right)=\phi\left(|D|^{2}\right) \sum_{k} \frac{1}{k^{2}}<\infty .
$$

This implies that for each $\epsilon>0$ there is a projection $w \in \mathcal{M}$ such that

$$
\left\|\left(\frac{1}{k} \alpha^{k} D\right) w\right\|_{\infty} \rightarrow 0 \quad \text { and } \quad \phi(1-w)<\frac{\epsilon}{4}
$$

cf. 8.

By Lemma 2.6, there exists a projection $r \in \mathcal{M}$ with $\phi(1-r)<\frac{\epsilon}{4}$ such that

$$
\left\|\frac{D}{k} r\right\|_{\infty} \rightarrow 0
$$

Summing up, for a given $\epsilon>0$, we have

$$
A-\tilde{A}=D-\alpha D+b+d \quad \text { with } \quad D, b, d \text { as above. }
$$

Let us put $\beta=\sum_{i=1}^{4}\left(b_{i}+d_{i}\right)$. By Lemma 2.5 there is a projection $q$ such that $\phi(1-q)<\frac{\epsilon}{2}$ and $\left\|q S_{k}(\beta) q\right\|_{\infty}<\frac{\epsilon}{2}(k=1,2, \ldots)$.

Thus, finally, taking $p=q \vee r \vee w$ we can say that for $\epsilon>0$ there is a projection $p \in \mathcal{M}$ such that

$$
\left\|p\left(S_{k}(A)-\tilde{A}\right) p\right\|_{\infty}<\epsilon, \quad \text { for } k>k_{0} \text { and } \phi(1-p)<\epsilon .
$$

By $(6)$ we find a sequence $\left(p_{n}\right)$ of projections and a sequence $\left(m_{n}\right)$ of positive integers such that

$$
\phi\left(1-p_{n}\right)<2^{-n} \epsilon \quad \text { and } \quad\left\|p_{n}\left(S_{k}(A)-\tilde{A}\right) p_{n}\right\|<2^{-n} \epsilon, \text { for } k>m_{n} .
$$

Putting $q=\bigwedge_{n \geq 1} p_{n}$, we get

$$
\phi(1-q)<\epsilon \quad \text { and } \quad\left\|q\left(S_{k}(A)-\tilde{A}\right) q\right\|_{\infty} \rightarrow 0 \quad \text { as } k \rightarrow \infty .
$$

Again, by Lemma 2.6,

$$
\left\|S_{k}(A)(\omega)-\tilde{A}(\omega)\right\| \rightarrow 0 \quad \mu \text {-a.e., }
$$

which ends the proof of the theorem.

\section{Comments}

By a slight modification of the above proof we can obtain a more general result.

3.1. Theorem. Let $A$ be a finite von Neumann algebra with a faithful normal trace $\tau$. Let $\alpha$ be a positive unitary map of $L_{2}(A, \tau)$ satisfying the condition

(7) $\frac{1}{n} \sum_{k=-n}^{n} \alpha^{k} \mathbf{1} \leq Y \quad(k=1,2, \ldots)$

$$
\text { for some } Y \text { affiliated to } A \text { with } \tau(Y)=\int_{0}^{\infty} \lambda \tau(e(d \lambda))<\infty \text {. }
$$

Then $S_{n}(x)=\frac{1}{n} \sum_{k=0}^{n-1} \alpha^{k}(x) \rightarrow \tilde{x}$ almost uniformly, for each $x \in L_{2}(A, \tau)$.

We formulated Theorem 1.2 as the main result because our intention was to prove an elementary fact on random matrices of second order. It would be interesting to find its direct proof without any reference to the operator algebras. 
3.2. Comparing both theorems with the classical results [6], [10], [5], one can suspect that the assumption (2) is superfluous, or, maybe, assuming (2) we can drop the condition of positivity. Unfortunately, we did not manage to answer this question. Our conjecture is that in the noncommutative case of $\mathcal{H}$, the positivity of $\alpha$ does not imply (4) without any additional condition (maybe weaker, then (2)).

3.3. Denote by $\mathcal{H}_{0}$ the subspace of $\mathcal{H}$ consisting of diagonal matrices. If $\alpha\left(\mathcal{H}_{0}\right) \subseteq$ $\mathcal{H}_{0}$ and $\alpha$ is positive, then $\alpha$ satisfies (2). Indeed, $\mathcal{H}_{0}$ can be identified with the Hilbert space $\mathcal{K}$ of vectors $f=\left(f_{1}, \ldots, f_{d}\right), f_{j} \in L_{2}(\Omega, \mu)$, with the inner product $(f, g)=\int_{\Omega}\left(\sum_{j=1}^{d} f_{j} \bar{g}_{j}\right) d u$. The unitary map $\alpha$ induces a positive isometry $\tilde{\alpha}$ in $\mathcal{K}$ (obviously, $f \geq 0$ means that $f_{i} \geq 0$, for $i=1, \ldots, d$ ). By the commutativity of $\mathcal{H}_{0}$ we can easily adopt the classical argument to get the following maximal ergodic inequality.

For $f \geq 0, \sup _{n} \frac{1}{n} \sum_{k=0}^{n-1} \tilde{\alpha}^{k} f \in \mathcal{K}$ (cf. 2] or [11, p. 87), which gives (2) for $f=(1, \ldots, 1)$.

3.4. Let us note that if $\alpha$ is a positive isometry in $\mathcal{H}$ satisfying $\alpha \mathbf{1}=\mathbf{1}$, then (4) follows immediately from Yeadon's ergodic theorem ([13], 7]).

\section{REFERENCES}

[1] Charn Huen Kan, Ergodic properties of Lamperti operators, Canad. J. Math. 30 (1978), 1206-1214. MR 80g:47037

[2] A. de la Torre, A simple proof of the maximal ergodic theorem, Canad. J. Math. 28 (1976), 1073-1075. MR 54:5867

[3] J. Dixmier, Formes linéaires sur un anneau d'opérateurs, Bull. Soc. Math. France 81 (1953), 9-39. MR 15:539a

[4] R. Duncan, Some pointwise convergence results in $L^{p}(\mu), 1<p<\infty$, Canad. Math. Bull. 20 (1977), 277-284. MR 58:17038

[5] R. Duncan, Pointwise convergence theorems for self-adjoint and unitary contractions, Ann. Prob. 5 (1977), 622-626. MR 56:3247

[6] A. Ionescu Tulcea, Ergodic properties of isometries in $L^{p}$ spaces, $1<p<\infty$, Bull. Amer. Math. Soc. 70 (1964), 366-371. MR 34:6026

[7] R. Jajte, Strong Limit Theorems in Noncommutative Probability, Lecture Notes in Math. 1110, Springer-Verlag, Berlin-Heidelberg-New York-Tokyo, 1985. MR 86e:46058

[8] R. Jajte, Strong Limit Theorems in Noncommutative $L^{2}$-spaces, Lecture Notes in Math. 1477, Springer-Verlag, Berlin-Heidelberg-New York-Tokyo, 1991. MR 92h:46091

[9] E. Nelson, Notes on non-commutative integration, J. Funct. Anal. 15 (1974), 103-116. MR 50:8102

[10] I. E. Segal, A non-commutative extension of abstract integration, Ann. Math. 57 (1953), 401-457; erratum, ibid. 58 (1953), 595-596. MR 14:991f MR 15:204h

[11] E. M. Stein, Topics in Harmonic Analysis Related to the Littlewood-Paley Theory, Ann. Math. Studies 63, Princeton University Press, Princeton, New Jersey, 1970. MR 40:6176

[12] S. Ştrătilă and L. Zsido, Lectures on von Neumann Algebras, Editura Academiei, Bucureşti and Abacus Press, Turnbridge Wells, 1979. MR 81j:46089

[13] F. J. Yeadon, Ergodic theorems for semifinite von Neumann algebras I, J. London Math. Soc. 2 (16) (1977), 326-332. MR 58:7111

Faculty of Mathematics, University of Łódź, Banacha 22, PL-90-238 Łódź, Poland

E-mail address: rjajte@math.uni.lodz.pl 